\title{
Topic Study Group No. 12: Teaching and Learning of Geometry (Primary Level)
}

\author{
Sinan Olkun, Ewa Swoboda, Paola Vighi, Yuan Yuan \\ and Bernd Wollring
}

\section{Introduction}

The aim of the TSG 12 working group is to promote the sharing of research on early geometrical thinking and understanding with a special focus on the kindergarten and primary education level.

The group provided a forum for discussion of the learning and teaching of geometry, from the historical, epistemological, cognitive, semiotic, and educational points of view, related to students' difficulties and to the design of teaching and curricula.

The topic study groups were organised in four sessions, each of them with 30-40 participants, with 4 invited lectures, 23 papers, and 2 posters.

During the working group, a main important suggestion emerged: The topic of geometry plays a limited role in early mathematical practices, while for young children geometric and spatial thinking could be fundamental in development in and beyond geometry.

Co-chairs: Sinan Olkun, Ewa Swoboda.

Team members: Paola Vighi, Yuan Yuan, Bernd Wollring.

\author{
S. Olkun $(\bowtie)$ \\ TED University, Ankara, Turkey \\ e-mail: sinanolkun@gmail.com; sinan.olkun@tedu.edu.tr \\ E. Swoboda \\ University of Rzeszów, Rzeszów, Poland \\ e-mail: eswoboda@ur.edu.pl \\ (C) The Author(s) 2017 \\ G. Kaiser (ed.), Proceedings of the 13th International Congress on Mathematical \\ Education, ICME-13 Monographs, DOI 10.1007/978-3-319-62597-3_39
}




\section{Description of TSG-12 Sessions}

\section{Recognition and Classification of Shapes}

The problem of identifying, defining, and classifying geometric shapes was the dominant issue in discussions. This issue is not a new one, but its implementation can be treated very differently. Still, many researchers believe that the ability to recognise shapes is one of the essential elements of early geometrical knowledge. This view does not arouse controversy, but the way it has been implemented in research varies. Much research has determined the current level of students' knowledge in this area, but more and more often didactical proposals have been presented that create an area for collecting of geometric intuition.

Clements and Sarama focused on the 3- to 8-year-old children's knowledge of shapes. They described a large-scale study that mainly dealt with a detailed analysis of children's responses to a set of shape identification tasks (related to typical shapes) using a wide variety of examples and non-examples. The main result obtained is that "pre-school-aged children can learn not only matching and naming shapes but also learn about their components and (some) properties".

However, researchers have devoted more attention to testing various proposals about how to build students' knowledge. Such ideas often take the form of a comprehensive long-term program in which many issues of geometrical education permeate. Often, these proposals are embedded in a rich learning environment. These studies also reveal new aspects of the knowledge of geometric figures in children's minds. The topic of "shape recognition" was the subject of the research presented by Coutat and Vendeira. They developed pre-geometric activities, starting from a collection of 75 shapes to manipulate, using didactical variables such as the number of sides, the convexity, the presence of straight or curved sides, and so on. They analysed the results in terms of "student's perception of the shape," "the use of characteristics," and "the use of a pertinent language," concluding that "manipulation helps the student in the use of characteristics to recognise shapes," promoting a progressive change in their visualisation. Another interesting proposal was presented by Vighi that connects the creation of knowledge of geometric figures with students' own creativity in the artistic environment. Activity was realised with children 5-6 years old, starting from a painting by Kandinsky and reproductions of it made by pupils. The main result involved the problem of manipulation of non-reversible shapes. Jirotková studies the mechanism of birth and development of geometrical schema in a pupil's mind. She presented three manipulative learning environments that contribute to building mental schemas of geometrical objects, relationships, and processes in a pupil's mind. 


\section{Definitions of Shapes}

Okazaki presented an analytical framework for the paths that students follow in constructing definitions, based on their understanding of inclusion among geometric figures. He found four kinds of understanding of inclusion relations: judgment based on visual characteristics, two common properties, relation between intensions and extensions, and genus-differentia definition.

Iskenderoğlu and Akgan developed a study to analyse the knowledge of definitions of two-dimensional geometrical concepts by prospective teachers, in which the definitions of twelve geometrical concepts was studied (angle, polygon, triangle, rectangle, trapezoid, parallelogram, rhombus, oblong, square, deltoid, and circle). The prospective teachers were struggling with the tests, often the definitions were not fully complete, possibly because of insufficient field knowledge. These results confirm that teacher preparation should be revised. This opens up a new research area on this issue.

Brunheira and Ponte analysed an exploratory task based on the hierarchical classification of quadrilaterals submitted to 30 prospective elementary teachers. The results highlighted the role of the constructions and negotiation of meanings. Gurhan and Zembat investigated the same topic, studying the main tenets of a technologysupported instructional sequence to foster a deep understanding of the hierarchy of quadrilaterals. Based on earlier work, argumentative activities in the study of triangle properties help students detect which properties are important in classifying geometric shapes and, consequently, constructing correct geometric concepts.

\section{Using Language}

Starting from the hypothesis that language can reveal some aspects of mathematical knowledge as well as their evolution, Guille-Biel Winder studied the learning of 6- to 7-year-old children during implementations of a situation involving reproduction of figure by folding. She analysed the results obtained from implementations of the PLIOX (a squared paper separated into four square, coloured zones) in two classrooms. An analysis of teachers' activities - language and gestures - with regard to three components (acting, talking, and thinking), in particular the use of language in the process of negotiating meaning, was the focus of the paper presented by Barrera-Curin, Bulf, and Venant. They compared the same mathematical situation in two different contexts (France and Québec). 


\section{Building and Representing 3D Shapes}

Research has shown that children's perceptions of different components of 3D geometric thinking are variable and complex. In particular, it requires the understanding of the relative locations of 3D objects to each other, the recognition of the properties of 3D objects, the relationship between a geometrical object and its visual representation, and the individuation of its properties starting from its drawings.

Reinhold and Wöller analysed the role of children's (aged 8-9) wooden block building activities in their conceptual knowledge of geometrical solids. In particular, they conducted interviews with children in Germany and Malaysia, and a qualitative analysis of the data shows a wide variety of individual activities. Denizli, Erdoğan, and Olkun developed a test to measure the 3D geometric thinking of first to fourth grade students and to investigate the development of $3 \mathrm{D}$ geometric thinking across the grades. In particular, they wanted to evaluate the component of "recognizing the properties of 3D objects". They showed that a student's ability to recognise these properties improves significantly with increasing grade level.

Issues related to 3D geometry reveal the problem of representation of these objects on a piece of paper (or computer screen). Studies have shown that this problem is nontrivial. Kondo presented problems of comparison of segment lengths and of angle amplitudes to students (aged 10-12) using 2D representations of cubes. The segments were edges or diagonals of a cube and the angles were identified by pairs of edges or by an edge and a diagonal. Yuan suggested that the use of virtual manipulatives creates an interactive environment to support multiple representations of geometric objects and to develop the ability of spatial structuring. Her study was focused on counting blocks in a 3D environment, which provided students with an initial understanding of spatial concepts. J.A. Cochran, Z. Cochran, and Hopper proposed activities on the transition from 2D to 3D objects using a new technology, 3D printing. Athias compared tools in the pen-and-pencil environment and dynamic geometry software with young pupils (aged 9-10), showing how a mathematical concept (equal length) could be used in the two environments.

\section{Mental Manipulation}

Generally, the problem of dynamic reasoning in geometry has been associated with intuitions of isometric transformations. However, the extension of the theoretical foundations of research in geometry has given another direction to research. It has become important to examine the extent to which children possess the ability to make mental transformations of objects and test didactical proposals, which gives an opportunity to gather experience in a dynamic interpretation of static images.

Swoboda and Zambrowska studied students' mental manipulation of a shape at the early educational level. They analysed the performance of students in one task 
with attention to dynamic thinking. Ramful and Lowrie also investigated mental manipulation (rotation) with students 11-13 years old through the design of a novel instrument. They concluded that spatial reasoning correlates with performance in mathematics. Jirotková presented manipulative environments in which specially created series of tasks enabled children to develop their competences in dynamic understanding of changes.

\section{Geometrical Relations}

Swoboda presented research among 4- to 7-year-old children on development of the "geometrical regularities" script. Additionally, she showed a linkage between the way 6-year-old children use geometric regularities and their later functioning at school. Kim and Kim explored the question "Does the convergent instructional model cultivate core competencies in the field of mathematics?" They concluded that esthetical designs can be thought to produce creative problem-solving ability. Vighi analysed the role of symmetry in a dynamic approach and the difficulties connected with its practice.

\section{Using Non-typical Tools that Support Presentation of Some Geometrical Concepts}

Olkun studied both numerical and geometric reasoning promoted by number-line estimation tasks. He concluded that the ability to estimate the relative magnitude of numbers on an empty number line has more to do with geometry achievement than arithmetic. Hassan Mohamed presented special tools that were realised with the aim of helping blind pupils to learn geometrical constructions. The results showed the effectiveness of these tools on the blind pupils' skills in this field. Iwase et al. studied mathematical knots, i.e., closed curves in space. They proposed and analysed some examples of teaching methods in elementary and junior high school.

Open Access Except where otherwise noted, this chapter is licensed under a Creative Commons Attribution 4.0 International License. To view a copy of this license, visit http://creativecommons. org/licenses/by/4.0/.

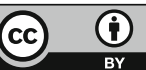

